\title{
Radio Frequency Ablation of Complex Arrhythmias Using Carto System
}

\section{R Yadav, N. Naik, R.Juneja, G Sharma, S Ramakrishnan, S Anandraja, KK Talwar.}

We present our initial experience with the use of CARTO system in mapping and ablation of complex arrhythmias. 33 patients (mean age $37 \pm 11.3$ years, 23 males) were Studied. Clinical arrhythmias were atrial flutter (AF) in 5, intra-atrial reentrant tachycardia (IART) in 7, ectopic atrial tachycardia (EAT) in 3, inappropriate sinus tachycardia in I, Arrhythmogenic right ventricular cardiomyopathy (ARVC) in 5, fascicular VT in 1, left ventricular outflow (LVOT) VT in 3, ischemic VT in 2, right ventricular outflow (RVOT) VT, in 1 and left, ventricular (LV) VT in 5 . There were 2 coronary artery disease, 5 ARVC, 1 each of dilated \& restrictive cardiomyopathy, atrial septal defect and mitral stenosis. Nine patients had congenital heart disease, while 3 patients of left ventricular tachycardia had Left ventricular hypertrophy (LVH). Ablation was successful in 4 patients with AF, 5 with IART, 2 with EAT (1 non inducible), all LVOT VT, 2 with LVVT, 4 with ARVC and one each in the fascicular and RVOT VT. One patient with ischemic VT, 2 with ARVC and 1 with LVOT had recurrence, Two patients developed an allergic response to the reference patch.

In conclusions, three dimensional mapping using CARTO system permits better understanding of tachycardia mechanism in complex cases and helps in guiding radio frequency ablation.

All India Institute of Medical Sciences, New Delhi. 